\title{
Traumatic Bulbar Urethral Stricture: Improvised Reconstruction with Muscle and Nerve Sparing Approach
}

\author{
Mukesh Chandra Arya ${ }^{*}$, Ankur Singhal ${ }^{2}$, Ajay Gandhi ${ }^{2}$, Yogendra Shyoran², Mahesh Sonwal' and Rakesh \\ Singh ${ }^{2}$
}

${ }^{1}$ Professor and Head, Department of urology, Sardar Patel medical college, Bikaner, Rajasthan, India.

${ }^{2}$ Department of urology, Sardar Patel medical college, Bikaner, Rajasthan, India.

*Corresponding author: Mukesh C Arya, Professor and Head, Department of urology, Sardar Patel medical college, Bikaner, Rajasthan, India.
Received Date: October 20, 2020

Published Date: November 13, 2020

\begin{abstract}
Introduction: Anastomotic urethroplasty in traumatic bulbar stricture is successful in 90-95\% of cases, but for post-void dribble and ejaculatory dysfunction (EjD). Here, we present modified muscle and nerve-sparing urethroplasty to overcome these problems.

Material and Methodology: A retrospective analysis (from January 2015- January 2019) of 55 patients with traumatic bulbar stricture managed by standard urethroplasty (Group 1, N=30) and modified urethroplasty (Group2, $\mathrm{N}=25$ ) was done. The comparison also included their post-operative EjD and post-void dribble.

Results: Mean age of patients was 31.12 (15-55) years. Mean length of stricture was 1.41 [1-2] cm. Mean Qmax was 27.8 and $26.4 \mathrm{ml} / \mathrm{s}$ in the modified and standard urethroplasty group (P-value>0.05). EjD was calculated for each patient using questions from male sexual health questionnaire (MSHQ)and MSHQ mean scores pertaining to ejaculation had a significant difference between Group1 and Group2 with mean postoperative scores of 14.17 and 21.12 respectively (p-value- <0.005) At 1-year, ten patients (33\%) from Group 1 while one patient (4\%) in the Group 2 showed post-void dribbling respectively (P-value-0.007).

Discussion: Success rate (patient not needing post-operative intervention) was $100 \%$ in modified and $96.66 \%$ in standard urethroplasty group. Results in terms of EjD and post-void dribble were statistically significant.

Conclusion: In traumatic bulbar stricture, muscle and nerve-sparing urethroplasty is associated with statistically significant better outcomes in terms of EjD and post-void dribble.
\end{abstract}

Keywords: Muscle-sparing urethroplasty; End to end urethroplasty; EPA; Traumatic bulbar stricture; Stricture urethra

Abbreviation: EjD: Ejaculatory dysfunction; Qmax: maximum urine flow rate; MSHQ: male sexual health questionnaire; Qmax: Maximum Urine Flow Rate; RGU: Retrograde Urethrogram; SPC: Suprapubic Cystostomy; MCU: Micturating Cystourethrogram; PGA: polyglycolic Acid; DVIU: Direct Visual Internal Urethrotomy

\section{Introduction}

Bulbar urethra is the most common site of the stricture (46.9\%). Meta-analysis of anterior urethral strictures showed etiology as iatrogenic (33\%), idiopathic (33\%) and, to a lesser extent, trauma (19\%) and inflammation (15\%). End-to-end anastomosis is the most valid treatment of choice for short bulbar traumatic urethral strictures, with cure rates close to $100 \%[1,2]$. Bulbospongiosus muscle which covers bulbar urethra is primarily responsible for last few drops of urine and semen expulsion. Ejaculatory dysfunction (EjD) and post-void dribbling are common postoperative complications after muscle cutting bulbar urethroplasty in $23.3 \%$ 
and $29 \%$ patients, respectively. Here, we used a modified technique of sparing muscle and nerve to avoid these sequelae.

\section{Material and Methodology}

A retrospective analysis of 55 patients (January 2015 to January 2019) with traumatic bulbar stricture was done. Patients with post-inflammatory or with prior catheterization (without trauma) were excluded from the study group. In our department, retrograde urethrogram (RGU) is not done at the time of trauma. Based on the history of perineal trauma with blood at meatus, a presumptive diagnosis of bulbar urethral injury was made and trocar Suprapubic Cystostomy (SPC) was done. RGU and micturating cystourethrogram (MCU)were done at 3 months after injury. In cases with normal urethrogram, the trauma was presumed to be contusion. Such patients who voided well after clamping SPC were excluded. Those who had complete or near obliterative stricture were taken up for this study. Patients were asked about their complaints of post-operative EjD during their follow-up using 5 questions from MSHI (Male Sexual health questionnaire score) pertaining to ejaculation including frequency, volume, force and bother and compared with their pre-operative scores. Also, patients were asked "If they wet their undergarments after passing urine" to ascertain post-void dribble. Statistical analysis between these groups was done using IBM SPSS 25.0. From January 2015 to February 2017, all 30 patients were operated by standard muscle cutting technique (Group 1) and later on, 25 patients by modified muscle and nerve-sparing technique (Group 2).

Surgical technique: Detailed history, blood investigations including complete blood count, serum creatinine and ultrasound abdomen was followed by RGU and MCU. Under anesthesia, antegrade and retrograde urethroscopy showed complete cut off at the level of the bulbar urethra. With the patient in the lithotomy position, midline perineal incision was given.

Standard technique: Bulbospongiosus muscle was divided in the midline. The bulbar urethra was mobilized up to the penoscrotal junction. Stricturous segment was identified and excised. A few interrupted $4 / 0$ chromic catgut sutures were taken radially to tack the mucosa to the urethral wall and prevent retraction during the anastomosis. Proximal and distal urethral segment were spatulated at 6'o clock and 12'o clock respectively. End to end anastomosis was done over 14 Fr Silastic Foleys catheter using 4-0 polyglycolic acid(PGA) and drain was put before layered closure. The drain was removed on post-operative day 2 and Foleys catheter on day 21.

Modified technique: The perineal branch of the pudendal nerve is damaged during dissection of bulbospongiosus muscle which cannot be restored by suturing the muscle. So bulbospongiosus muscle was carefully separated from the corpus spongiosum by sharp dissection leaving the lateral margins of the muscle and central tendon of perineum intact. To expose the bulbar urethra, the muscle was pulled down using two small right-angle retractors (Figure 1).

The scarred urethra was excised and both ends were spatulated as in the standard technique. The proximal urethral segment was sutured to corpora at 11,12 and 1'0 clock, followed by an end to end anastomosis. Ventrally thick corpus spongiosum was sutured in two layers from 3 - 9 o'clock with continuous 4-0 PGA suture over 14 F Silastic Foleys catheter (Figure 2).
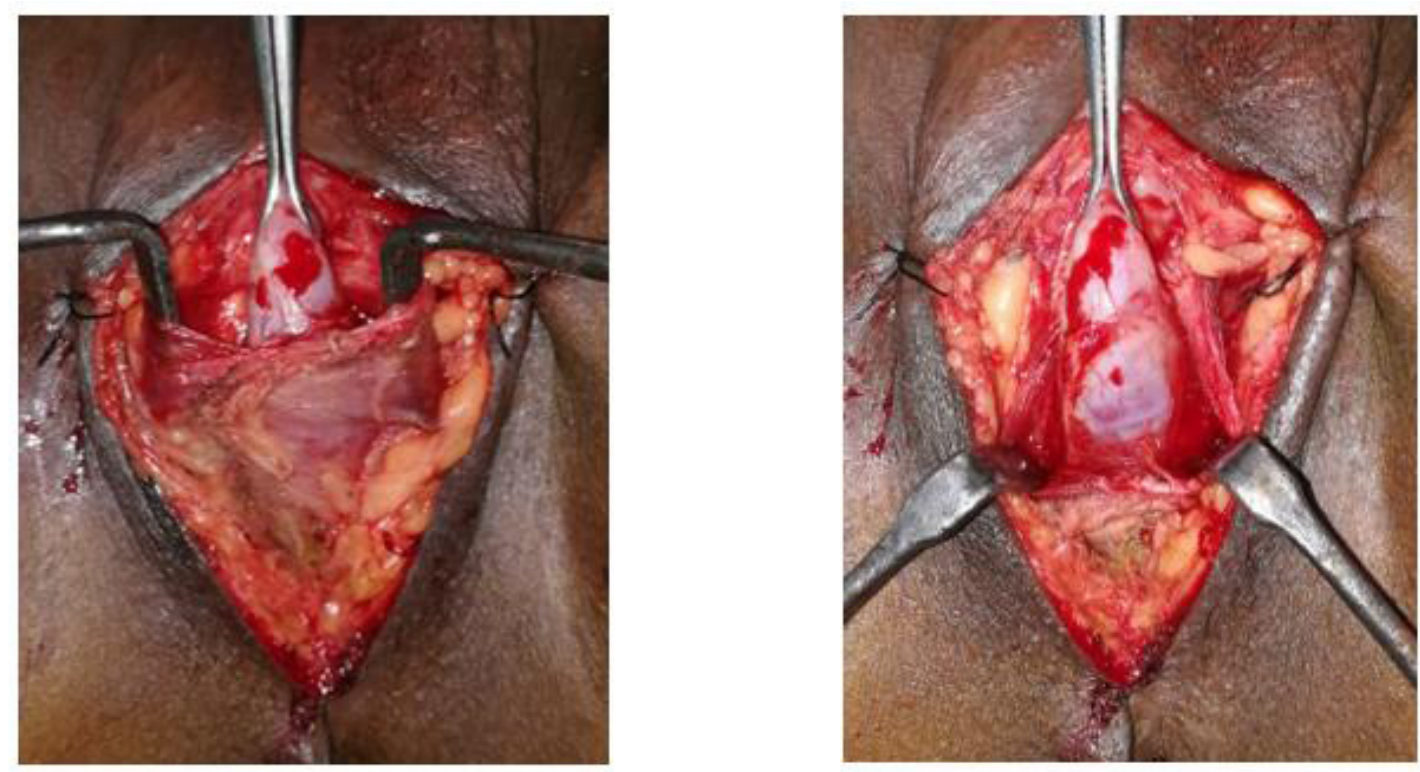

Figure 1: Muscle and nerve-sparing demonstrated. 

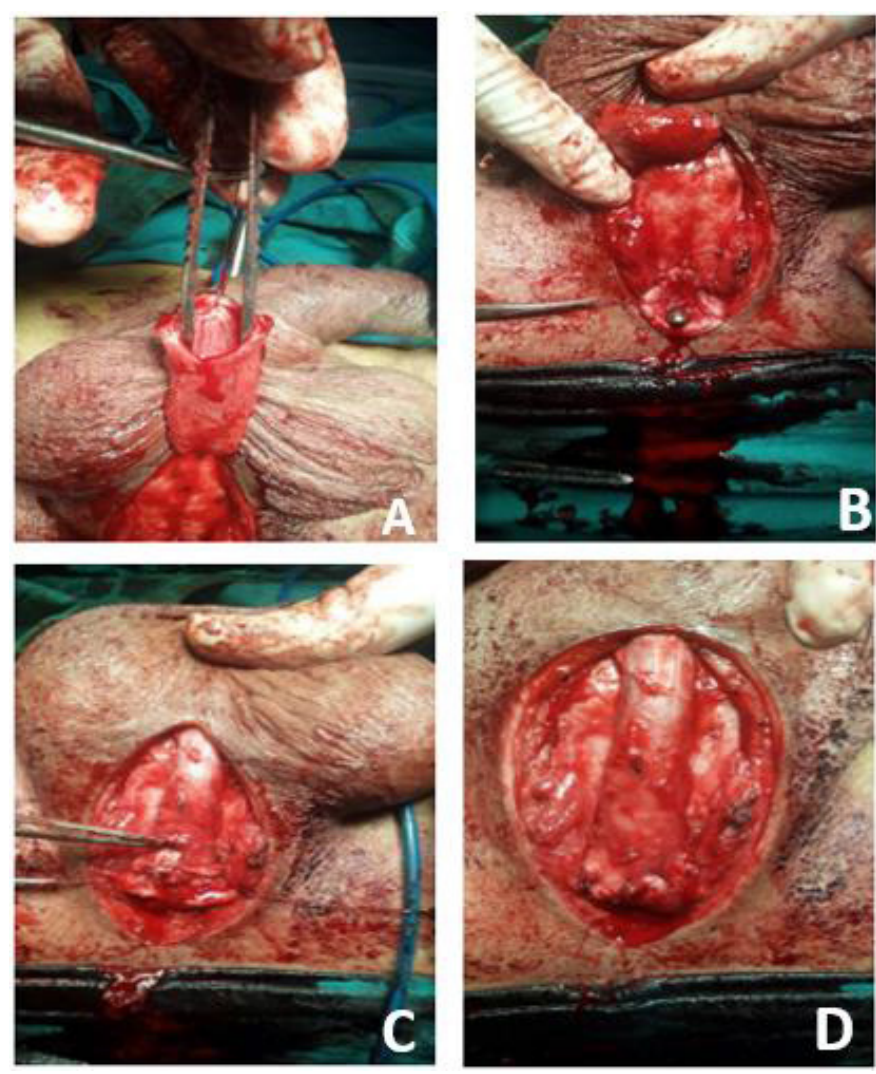

Figure 2: (A) 12 O' Clock Spatulation in Distal Urethra (B) Proximal Urethral Segment Sutured To Corpora At 11, 12 \& 1 O' Clock Positions With 4-0 Pga (C)First Layer Of Anastomosis Done With 4-0 Pga (D) Anastomosis Completed In Two Layers.

This establishes early vascular continuity in corpus spongiosum and achieves better healing. The closure was done as in the standard technique. Prophylactic 3rd generation cephalosporin and amikacin were given for 3 days. Postoperative uroflowmetry was done at 6 weeks and RGU was done at 6 months (for documentation) in all patients. Patients were followed up for a period of one-year and were asked about their EjD and post-void dribble. Failure was considered if the patient had symptoms post-operatively or required an ancillary procedure. The patient was considered cured in the absence of symptoms and not needed any intervention.

\section{Results}

Age: ranged from 11 to 55 years with the mean age 31.12 years (Table 1).

Table 1: Age at operation.

\begin{tabular}{|c|c|c|}
\hline Age (in years) & Num of Patients & Percentage \\
\hline $15-25$ & 22 & $40 \%$ \\
\hline $26-35$ & 18 & $32.70 \%$ \\
\hline $36-45$ & 8 & $14.54 \%$ \\
\hline $46-55$ & 7 & $12.70 \%$ \\
\hline
\end{tabular}

Presentation: One patient (Group 1) presented with periurethral abscess following catheterization after trauma. SPC and drainage of the abscess was done. Rest of the patients were managed with immediate SPC followed by MCU and repair at three months.

Duration: The mean duration of surgery was 65 (54-74) minutes in Group 1 and 70 (58- 84) minutes in Group 2.

Length of stricture: Mean length of the stricture was $1.41 \mathrm{~cm}$ in our series. Length of the stricture was 1.47 and $1.36 \mathrm{~cm}$ in Group 1 and 2 respectively.

Postoperative uroflow: Uroflow was done at 6 weeks and overall Mean Qmax was $23.036 \mathrm{ml} / \mathrm{s}$ (Table 2).

Results: Success rate (patient not needing post-operative intervention) was $100 \%$ in modified and $96.66 \%$ in standard urethroplasty group.

Post-operative EjD: EjD was evaluated by asking patients questions about frequency, volume, force and bother related to their ejaculation. Questions were framed from MSHQ (MSHQ5, MSHQ7, MSHQ8, MSHQ9, MSHQ12). Group 1 and Group 2 patients had a significant difference in their score pertaining to EjD and P-value was highly significant (Table 3, Figure 3). 
Table 2: Post-operative Mean Qmax.

\begin{tabular}{|c|c|c|}
\hline Qmax (in ml/s) & Number of Patients & Mean Qmax (ml/s) \\
\hline Modified urethroplasty & 25 & 23.8 \\
\hline Standard urethroplasty & 30 & 22.4 \\
\hline Total & 55 & 23.036 \\
\hline
\end{tabular}

Post- void dribbling: Post-void dribble was seen in 10 patients $(33.3 \%)$ in the standard group and 1 patient (4\%) in the modified group (P-value 0.007).

Complications: None of the patients had a fistula. Only 1 patient $(3.33 \%)$ with a prior history of catheterization and periurethral abscess had a stricture recurrence in the standard group. The success rate for our technique was $100 \%$ in this small series of patients operated by the modified technique. No patient had any intraoperative complications or required blood transfusion.

Wound infection: Three patients (10\%) in Group 1 and two patients (8\%) in Group 2 had wound infection respectively. All were managed conservatively with success. P-value $(0.797)$ was not significant.

Table 3: Post-operative EjD.

\begin{tabular}{|c|c|c|c|c|c|c|}
\hline S. No. & Questionnaire & $\begin{array}{c}\text { Group 1 Pre-Trauma } \\
\text { Mean Score }\end{array}$ & $\begin{array}{c}\text { Group 1 Post-Trauma } \\
\text { Mean Score }\end{array}$ & $\begin{array}{c}\text { Group 2 Pre-Trauma } \\
\text { Mean Score }\end{array}$ & $\begin{array}{c}\text { Group 2 Mean Post-Trauma } \\
\text { Score }\end{array}$ & 4.32 \\
\hline 1. & MSHQ5 & 4.79 & 2.9 & 4.91 & 4.12 & $<.005$ \\
\hline 2. & MSHQ7 & 4.85 & 2.8 & 4.87 & 4.16 & $<.005$ \\
\hline 3. & MSHQ8 & 4.89 & 3.4 & 4.91 & 4.24 \\
\hline 4. & MSHQ9 & 4.82 & 2.2 & 4.83 & 4.28 & $<.004$ \\
\hline 5. & MSHQ12 & 4.78 & 2.87 & 4.79 & $<.005$ \\
\hline 6. & MSHQavg & 24.25 & 14.17 & 24.33 & 21.12 & $<.005$ \\
\hline
\end{tabular}

*2 patients in Group 1 and 1 patient in Group 2 were not able to answer the questionnaire as they were too young and had no prior experience of Ejaculation.

*MSHQ- Male Sexual Health Questionnaire

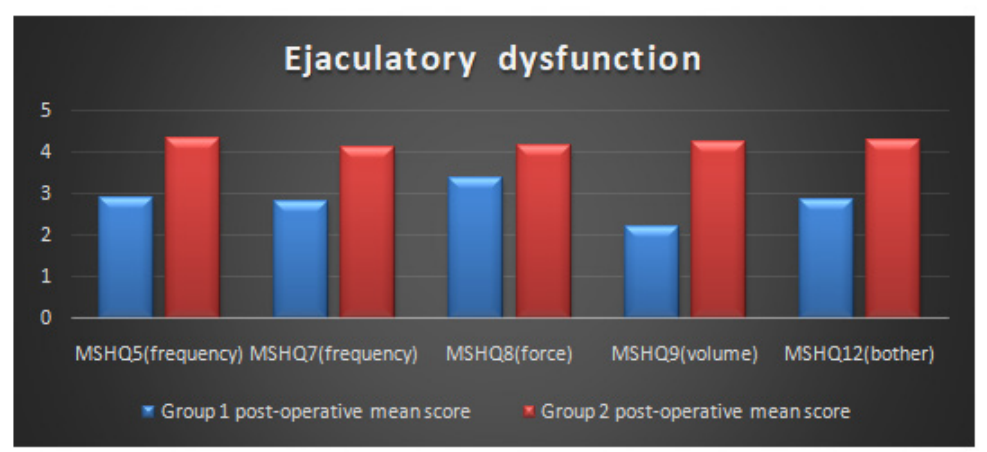

Figure 3: Post-operative EjD.

\section{Discussion}

Traumatic bulbar urethral strictures are usually of short length associated with intense spongiofibrosis. Therefore, such strictures are rationally managed with anastomotic urethroplasty rather than direct visual internal urethrotomy (DVIU) or augmentation. Immediate SPC, with delayed perineal anastomotic urethroplasty, remains the gold standard treatment. Bulbospongiosus is a paired muscle that covers the bulbar urethra. The rhythmic contractions of these muscles are responsible for the expulsion of semen and urine especially the last few drops. The perineal nerve (a branch of the pudendal nerve) innervates bulbospongiosus and ischiocavernosus muscles. Ejaculation may be disturbed by damage to the nerve and may result in a low volume of semen $[10,11]$. This nerve is damaged during dissection of bulbospongiosus muscles which can't be restored by approximating the muscles in midline. The success of urethroplasty is not only related to stricture recurrence but also to the impact on the erectile and EjD in addition to postvoid dribbling. Muscle sparing urethroplasty technique was initially described by Barbagli et al as a modification of the standard technique to decrease the occurrence of these complications [12]. The bulbar urethra is elastic and can be mobilized from its attachment allowing to bridge a gap of $2-3 \mathrm{~cm}$ at the stricture site. Additionally, $1 \mathrm{~cm}$ of this length is lost in spatulation of proximal and distal urethral segments on either side. The bulbous urethra also offers other characteristics that make it particularly suitable for urethroplasty:(i) the mucosa is well-differentiated and more abundant than the spongiosa, so can be anastomosed on two planes, i.e. mucosa-mucosa 
and spongiosa-spongiosa; (ii) the spongiosa is thick and wellvascularized, with an adequate blood supply. This reduces the risk of ischemia and secondary fibrosis, limiting the likelihood of recurrent stricture $[9,13]$. Moreover, postvoid dribbling and semen sequestration will likely be post-operative complications following any bulbar urethroplasty. EjD was described as difficult, slow seminal ejaculation or poor semen volume observed by the patient. Unmarried patients experienced EjD during masturbation. EjD and postvoid dribbling are very annoying complaints requiring manual compression of the bulbar urethra and compromising their quality of life despite the success of urethroplasty. Modified Bulbar urethroplasty with muscle and nerve-sparing aims to preserve these functions in a significant number of cases. The three-suture technique keeps the anastomosis open and reduces the recurrence of stricture. Two-layered closure of ventral corpus spongiosum establishes better blood supply and hence, sound healing. Most astonishingly on post-operative RGU, we were not able to localize the site of anastomosis due to these modifications (Figure 4- 6).
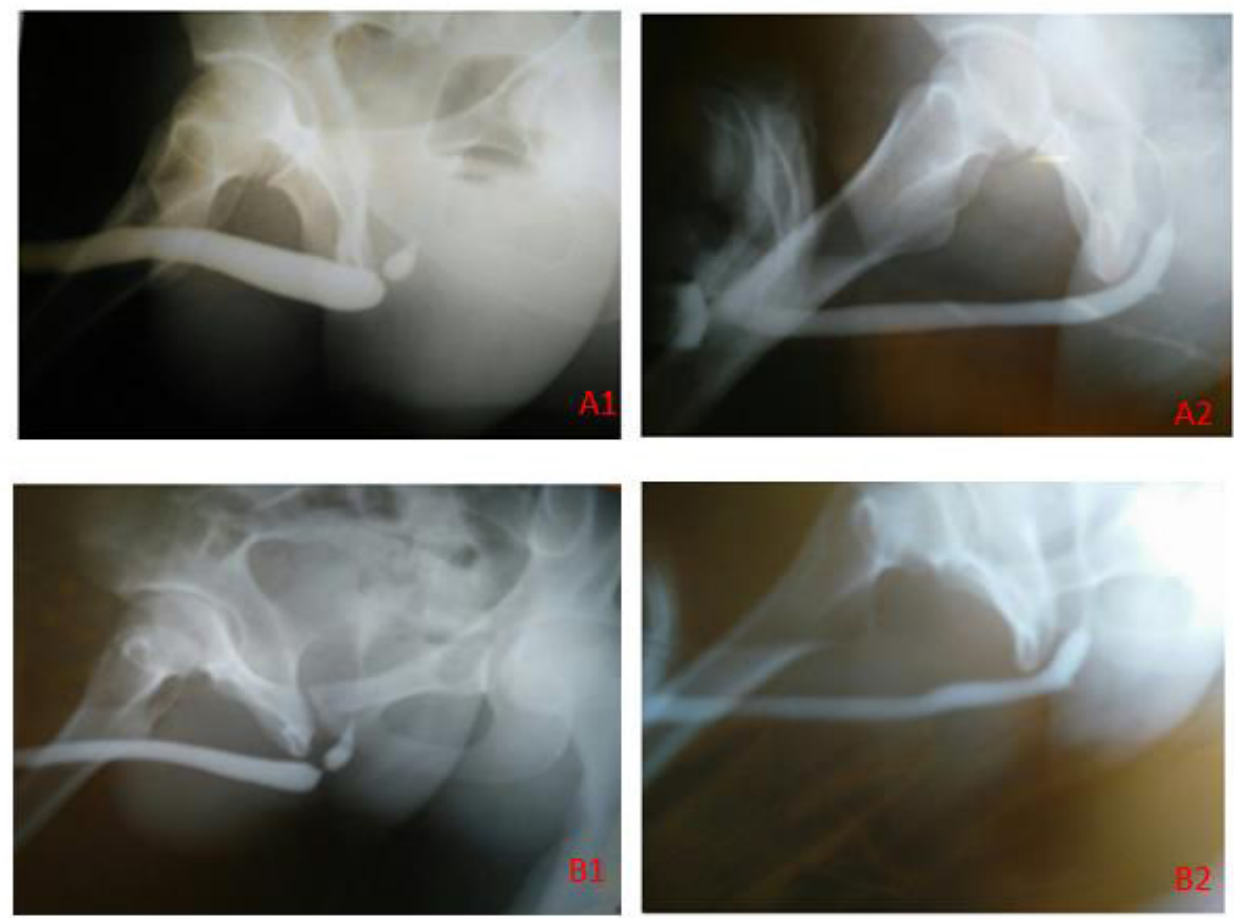

Figure 4: Patients treated by modified urethroplasty techniques

A1. PRE-OPERATIVE RGU

A2. POST-OPERATIVE RGU

B1. PRE-OPERATIVE RGU

B2. POST-OPERATIVE RGU
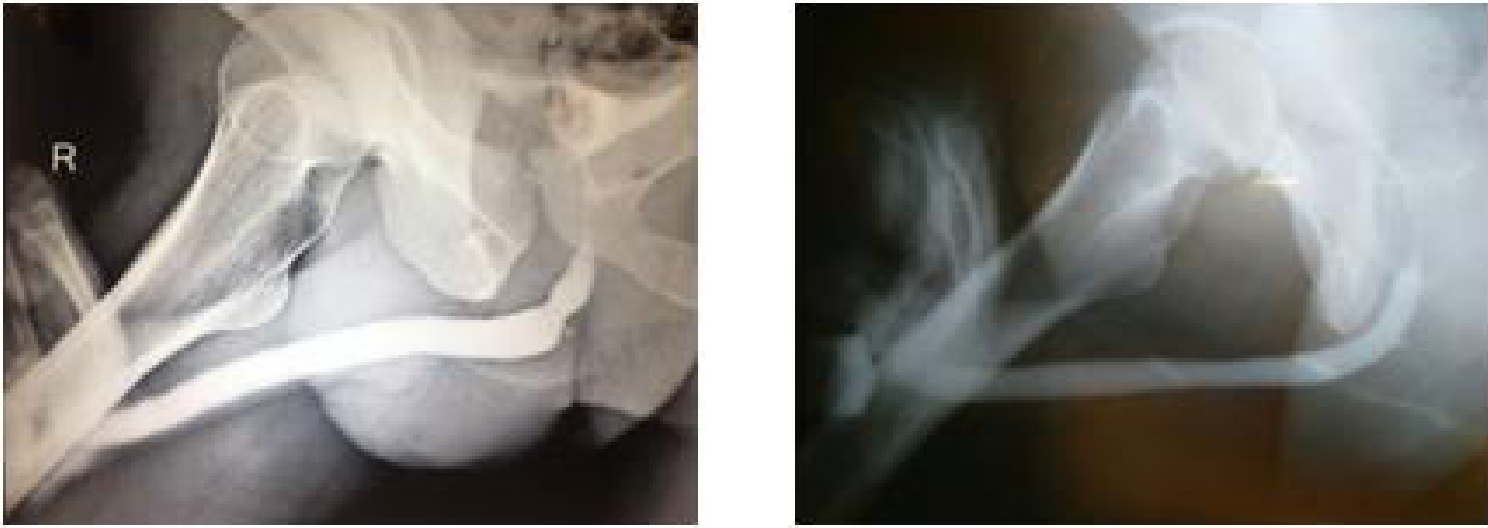

Figure 5: Comparison of post-operative RGU A) Standard Technique B) Modified Technique. 

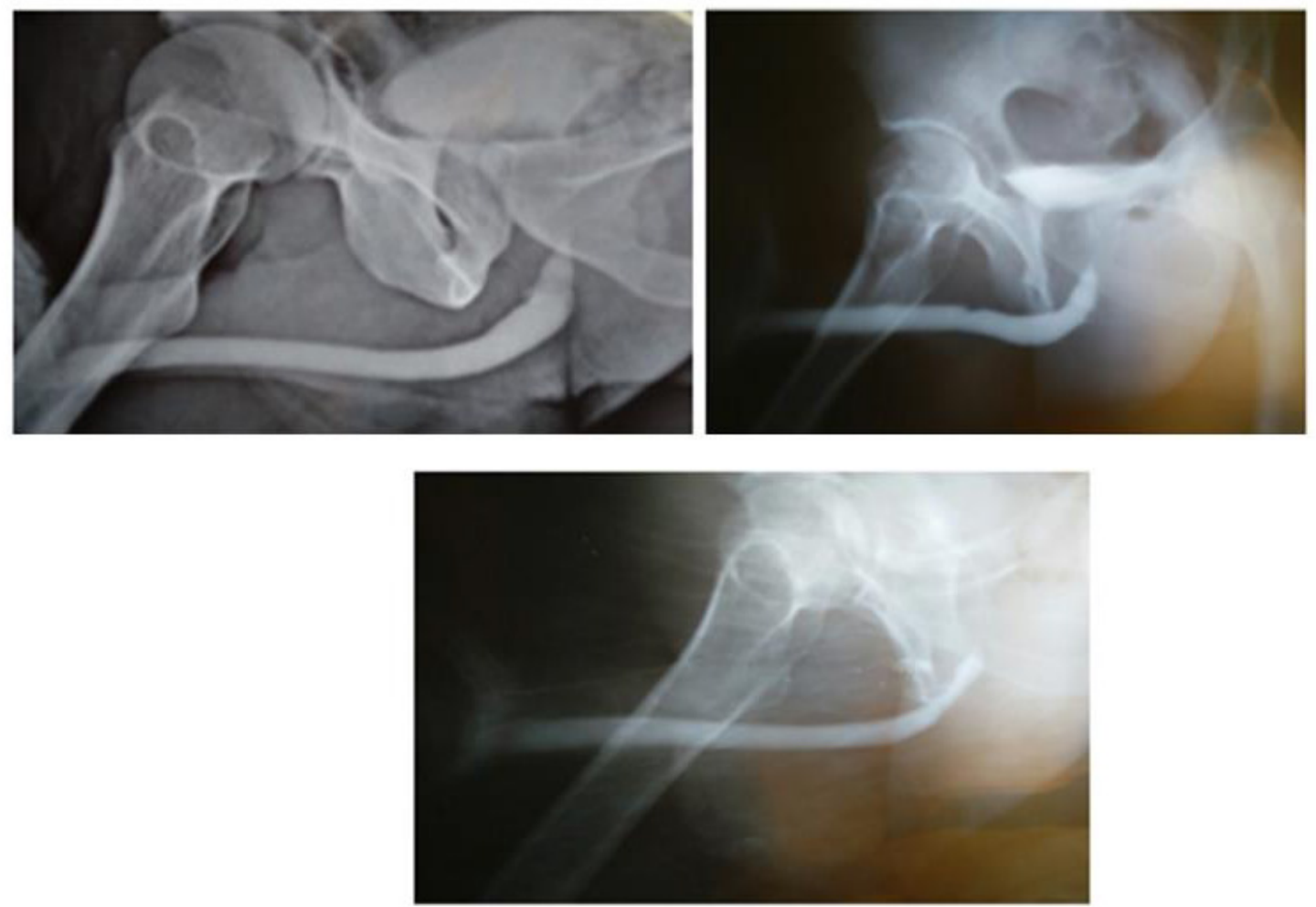

Figure 6: Post-operative RGU of other patients treated by a modified end to end urethroplasty technique.

Preservation of bulbospongiosus muscles and perineal nerves is advisable as they are involved in the expulsion of urine and semen from the bulb. The main limitation of end to end urethroplasty is the length of the stricture. If tried to bridge a longer gap, it may result in ventral curvature of the penis, and the anastomosis will be under tension with risk of failure [14]. In our series, stricture length ranged from 1 to $2 \mathrm{~cm}$ (mean-1.42 cm) and surgery outcomes were successful in $100 \%$ and $96.66 \%$ with Group 2 and 1 respectively. Similarly, Jun-Gyosuh et al. [15] found no recurrences in his series of 18 cases of end to end anastomoses. Elgammal MA [16] had $96 \%$ success rate in 24 out of 25 patients in post-traumatic bulbar urethral strictures. The age at presentation in this study ranged from 11 to 57 years with the mean age 31.4 years. the mean Qmax in our study was $23.04 \mathrm{ml} / \mathrm{s}$ while in the study by lumen et al. mean postoperative Qmax was $26.9 \mathrm{~mL} / \mathrm{s}$. Most of his patients were between 15 - 25 years. The results in our study confirm that patient age is not a factor in the success of the procedure and even old patients should not be denied surgery as observed by Barbagli et al. [17]. In our study, 2 patients had postvoid dribbling in Group 2 while no postvoid dribbling or semen sequestration was demonstrated in another study by Barbagli et al. [12]. Elkady E et al. [18]. also found that nine (36\%) and 1 patient (4\%) from muscle cutting and muscle-sparing group complained of postvoid dribbling with statistically significant difference between the two groups respectively $(\mathrm{P}=.01)$. EjD was evaluated by asking patients questions about frequency, volume, force and bother related to their ejaculation. Questions were framed from MSHQ (MSHQ5, MSHQ7, MSHQ8, MSHQ9, MSHQ12). Group 1 and Group 2 patients had a significant difference in their score pertaining to EjD and P-value was highly significant. Patients had a significant difference in each question of MSHQ related to ejaculation (frequency, volume, force and bother; p-value<0.005). Ariel Fredrick et al. [19] did a comparative study between muscle cutting and muscle-sparing urethroplasty (25 cases in each arm) for non-obliterative bulbar urethral stricture. They evaluated their results in terms of EjD and post-void dribble and found no significant difference. The plausible reason is that a significant number of their patients had preoperative EjD and post-void dribble in each arm due to stricture and these symptoms rather improved after surgery. Therefore, this study is not comparable to ours. We in our study did not come across any recurrence over a follow-up period of one year in the modified group, however1 patient had a recurrence in the standard group while in the study of Barbagli et al. [12], no patient had stricture recurrence. He had a history of catheterization after trauma and periurethral abscess. This resulted in long segment stricture and significant periurethral fibrosis. In such cases, muscle-sparing urethroplasty is not possible. Any attempt at catheterization in such patients can convert sterile hematoma into abscess and tissue loss. Prolonged catheterization may cause longer or even pan urethral stricture, making subsequent reconstruction difficult. This signifies the importance of urinary diversion rather than catheterization after urethral injury. This recurrent stricture was managed by 
redo-urethroplasty at 6 months. However, Elkady E. et al. found $3(12 \%)$ and $2(8 \%)$ recurrences in his study from muscle cutting and muscle-sparing group.

\section{Conclusion}

Standard anastomotic urethroplasty for traumatic bulbar stricture has a high success rate. However, a significant number of cases have bothersome post-void dribble and EjD despite successful urethroplasty. The three-suture technique with two-layered ventral spongiosal anastomosis resulted in indistinguishable suture line in post-operative RGU. Muscle and nerve-sparing technique showed statistically significant better results in terms of EjD and post-void dribble. This approach is, therefore, recommended in all cases of traumatic or inflammatory bulbar urethroplasties [17]. Patients with urethral injury are best served by a suprapubic diversion of urine and urethral rest rather than urethral catheterization.

Limitations: This is a retrospective study withoutrandomization.

\section{Acknowledgment}

None.

\section{Conflict of Interest}

No conflict of interest.

\section{References}

1. Berger AP, Deibl M, Bartsch G, Steiner H, Varkarakis J, et al. (2005) A comparison of one stage procedures for post-traumatic urethral stricture repair. Br J Urol 95(9): 1299-1302.

2. Koraitim MM (1995) The lessons of 145 posttraumatic posterior urethral strictures treated in 17 years. J Urol 153(1): 63-66.

3. Joshi P, Kaya C, Kulkarni S (2016) Approach to bulbar urethral strictures: Which technique and when. Turk J Urol 42(2): 53-59.

4. Hampson LA, McAninch JW, Breyer BN (2014) Male urethral strictures and their management. Nat Rev Urol 11(1): 43-50.

5. Bagchi, Puskal Kumar (2020) An audit of management of male urethral stricture and it's outcome: a single centre retrospective review. International Surgery Journal 7(3): 774-780.
6. Walsh PC, Retik AB, Vaughan ED Jr (2002) Campbell's urology. (9th ed.) Philadealphia: W.B. Saunders.

7. Webster GD, Mac Diarmid SA (1993) Posterior urethral reconstruction. In: Webster G, Kirly R, King L, Goldwasser B, editors. Reconstructive urology. Boston: Blackwell Scientific pp. 687-702.

8. Barbagli G, Bandini M, Balò S, Sansalone S, Butnaru D, et al. (2020) Surgical treatment of bulbar urethral strictures: tips and tricks. Int Braz J Urol 46(4): 511-518.

9. Lumen N, Spiers S, Backer De, Pieters R, Oosterlinck W (2011) Assessment of the short-term functional outcome after urethroplasty: a prospective analysis. Int braz j urol 37(6): 712-718.

10. Blaschko SD, Sanford MT, Cinman NM, Mc Aninch JW, Breyer BN (2013) De novo erectile dysfunction after anterior urethroplasty: a systematic review and meta-analysis. BJU Int 112(5): 655-663.

11. Yucel S, Baskin LS (2003) Neuroanatomy of the male urethra and perineum. BJU Int 92(6): 624-630.

12. Barbagli G, De Stefani S, Annino F, De Carne C, Bianchi G (2008) Muscleand nerve-sparing bulbar urethroplasty: a new technique. Eur Urol 54(2): 335-343.

13. Sobotta Anatomy (2018) Textbook 1st Edition: Friedrich Paulsen Tobias M Böckers Jens Waschke Published p.363.

14. MichelI E, Ranieri A, Peracchia G, Lembo A (2002) End-to-end urethroplasty: long-term results. BJU International 90(1): 68-71.

15. Suh JG, Choi WS, Paick JS, Kim SW (2013) Surgical Outcome of Excision and End-to-End Anastomosis for Bulbar Urethral Stricture. Korean J Urol 54(7): 442-447.

16. Elgammal MA (2009) Straddle injuries to the bulbar urethra: management and outcome in 53 patients. Int Braz J Urol 35(4): 450-458.

17. Barbagli G, De Angelis M, Romano G, Lazzeri M (2007) Long-term followup of bulbar end-to-end anastomosis: a retrospective analysis of 153 patients in a single center experience. J Urol 178(6): 2470-2473.

18. Elkady E, Dawod T, Teleb M, Shabana W (2019) Bulbospongiosus Muscle Sparing Urethroplasty Versus Standard Urethroplasty: A Comparative Study. Urology 126: 217-221.

19. Fredrick A, Erickson BA, Stensland K, Vanni AJ (2017) Functional Effects of Bulbospongiosus Muscle Sparing on Ejaculatory Function and PostVoid Dribbling after Bulbar Urethroplasty. J Urol 197(3 pt 1): 738-743. 